\title{
BMJ Open Chinese observational prospective study of ageing population with chronic kidney disease (C-OPTION): a study protocol
}

Shuang Liang, Wen-Ling Wang, Fang-Lei Zhu, Shu-Wei Duan, Xue-Feng Sun, Xiang-Mei Chen, Guang-Yan Cai, On behalf of Chinese observational prospective study of ageing population with chronic kidney disease (C-OPTION)

To cite: Liang S, Wang W-L, Zhu F-L, et al. Chinese observational prospective study of ageing population with chronic kidney disease (C-OPTION): a study protocol. BMJ Open 2018;8:e019457. doi:10.1136/ bmjopen-2017-019457

- Prepublication history for this paper is available online. To view these files, please visit the journal online (http://dx.doi. org/10.1136/bmjopen-2017019457)

Received 3 September 2017 Revised 21 December 2017 Accepted 4 January 2018
Check for updates

Department of Nephrology, Chinese PLA General Hospital, Chinese PLA Institute of Nephrology, State Key Laboratory of Kidney Diseases, National Clinical Research Center for Kidney Diseases, Beijing Key Laboratory of Kidney Disease., Beijing, China

Correspondence to Dr Guang-Yan Cai; caiguangyan@sina.com

\section{ABSTRACT}

Introduction The proportion of elderly people is steadily rising worldwide, especially in low-income and middleincome countries, including China. Chronic kidney disease (CKD) is a common disorder in older people. However, little is known about the epidemiology of CKD and its consequences among the elderly. Improvements on clinical guidelines and healthcare policies for this population are required. This study aims to examine the risk factors for progression of CKD among the elderly and develop models to identify subgroups who are at high risk.

Methods and analysis This is a prospective, multicentre, cohort study. The study population comprises $\sim 3000$ patients with predialysis CKD, aged $\geq 65$ years, recruited between March 2016 and December 2017. After the baseline assessments, these patients will be followed for 5 years or until the occurrence of primary outcomes. Assessments that include anthropomorphic measures, laboratory tests, questionnaires, and blood and urine specimen collection will be performed at baseline and at follow-ups. Data on demographic information, cognitive function, depression, risk of malnutrition, physical activity and quality of life will be collected. The primary outcomes are incidence of end-stage renal disease, loss of renal function ( $\geq 40 \%$ decline in glomerular filtration rate from baseline), and death. The secondary outcomes are acute coronary syndrome, hospitalisation for heart failure or unstable angina, cerebrovascular events, and peripheral arterial disease.

Ethics and dissemination This study protocol has been approved by the ethics committees of the Chinese People's Liberation Army General Hospital and the participating centres. All the participants gave written informed consent before data collection. The findings of the study will be published in peer-reviewed journals and will be presented at national or international conferences.

Trial registration number NCT03246204; Pre-results.

\section{INTRODUCTION}

The percentage of people who are elderly is increasing worldwide. By 2050, the population older than 60 years is expected to double, from $11 \%$ to $22 \% .^{1}$ This phenomenon is
Strengths and limitations of this study

- This is a prospective study design with an ethnically diverse, large-scale elderly population with chronic kidney disease (CKD) across China.

- A broad range of candidate risk factors will be monitored, especially non-disease-specific problems that are common in the elderly but are not traditionally considered.

- A limitation is that we recruited subjects based on a single measure of serum creatinine and/or albuminuria without a 3-month interval.

- There is no uniformed, standardised creatinine assay, and the CKD-Epidemiology Collaboration equation remains imperfect in elderly persons, as it is affected by loss of muscle mass.

- Some elderly patients with CKD are excluded from the study (eg, because of inability to comply), and therefore the results of the study may not be completely generalisable to all elderly patients with CKD.

also true of countries with populations that are predominantly of low income and middle income. ${ }^{2}$ In China in 2010, the number of people 60 years or older had already reached 177 million, according to the national census. ${ }^{3}$ An ageing population has important implications for medical care and public health systems.

Chronic kidney disease (CKD), characterised by poor health outcomes and high healthcare costs, is very common among the elderly. The National Health and Nutrition Examination Survey showed that the prevalence of $\mathrm{CKD}$ is $38 \%$ among those aged $>65$ years, compared with $13 \%$ of the overall US population. ${ }^{4}$

Risk modification in elderly patients with CKD is often challenging because of the heterogeneity of disease and the competing risks of other adverse events. ${ }^{5}$ While younger 
patients with CKD may progress to end-stage renal disease (ESRD), elderly patients are more likely to suffer mortality than progress to ESRD. ${ }^{6}$ The condition of older adults with CKD is often complicated by comorbidities and their associated adverse outcomes, ${ }^{7-9}$ and the CKD population has a high prevalence of geriatric syndromes that include frailty, cognitive impairment and impaired mobility.

These findings suggest that clinical decisions regarding older patients cannot be based on either chronological age alone, or factors such as elevated creatinine and proteinuria traditionally used to monitor kidney function. More comprehensive management is required, covering medical, functional and psychosocial problems. However, there are very little data on the management of these domains and associated outcomes of elderly patients with $\mathrm{CKD}$, and the current clinical guidelines often focus on specific renal diseases, but fail to consider overtly the contribution and implication of non-disease-specific problems.

For these reasons, we established a national cohort of Chinese elderly patients with CKD to enable prospective observational studies. The goals are the following:

1. To complete an epidemiological survey of CKD in the elderly across China, and evaluate the prevalence, distribution, aetiology and comorbidity patterns of CKD in this population.

2. To conduct a comprehensive geriatric assessment among older patients with CKD, including cognitive function, depressive symptoms, diet and nutrition, functional status, and quality of life. We aim to determine the prevalence of these non-disease-specific problems of older adults with CKD and to investigate their impact on outcomes.

3. To explore both traditional and non-traditional risk factors that are associated with adverse clinical outcomes. Traditional risk factors are those that have been well studied in renal disease, such as blood pressure and proteinuria. Non-traditional risk factors are more common among the elderly, but have been little considered in renal disease. Identifying these factors should aid the development of predictive models of subgroups of older patients with CKD who are at high risk.

\section{METHODS AND ANALYSIS}

\section{Study design}

This study is a multicentre, prospective cohort study of Chinese elderly with CKD, sponsored by the National Clinical Research Center for Kidney Disease at People's Liberation Army (PLA) General Hospital, located in Beijing, China. All of the participating clinical centres are renal departments at 34 hospitals, located in 26 cities in 24 provinces of China.

\section{Study population}

Recruitment of a baseline cohort of $\sim 3000$ participants was conducted between March 2016 and November 2017.
The participants will be followed up until the occurrence of a primary outcome or 5 years after enrolment.

The cohort of elderly patients is racially and ethnically diverse, with a broad spectrum of renal disease severity. All the participants conform to the following inclusion criteria: aged $\geq 65$ years old, received a diagnosis of CKD and provided signed informed consent.

We exclude participants if they (1) are participating in other interventional clinical trials; (2) had ESRD or receiving dialysis; (3) are diagnosed as having acute kidney injury; (4) had a history of renal or other transplantation; (5) had active malignancy within 24 months prior to screening, or metastatic cancer; (6) had a life expectancy less than 6 months; (7) had severe heart failure (New York Heart Association function class III or IV); (8) had HIV infection; (9) had isolated haematuria; and (10) were unable to communicate with examiners, unable to complete the study procedure even if assisted and otherwise unable to comply with the study protocol.

\section{Diagnostic criteria for CKD}

The diagnosis of CKD is based on the criteria of the 2012 Kidney Disease: Improving Global Outcomes, that is, a single measurement showing glomerular filtration rate (GFR) $<60 \mathrm{~mL} / \mathrm{min} / 1.73 \mathrm{~m}^{2}$ or proteinuria (albumin excretion rate $\geq 30 \mathrm{mg} /$ day or albumin to creatinine ratio (ACR) $\geq 30 \mathrm{mg} / \mathrm{g}),{ }^{10}$ or both.

The estimated GFR (eGFR, reported as $\mathrm{mL} /$ $\min / 1.73 \mathrm{~m}^{2}$ ) is calculated according to the serum creatinine ( $\mathrm{Scr}$ ) level obtained using either Jaffe's kinetic method or the Roche enzymatic method. If via Jaffe's kinetic method, the Modification of Diet in Renal Disease equation is adapted to data specific to Chinese patients with CKD: eGFR $\left(\mathrm{mL} / \mathrm{min} / 1.73 \mathrm{~m}^{2}\right)=175 \times \mathrm{Scr}^{-1.234}$ $\times \operatorname{Age}^{-0.179}(\times 0.79$, if female $)$, with age in years. ${ }^{11}$

Alternatively, if the Scr is measured by the Roche enzymatic method, the GFR is evaluated by the CKD-Epidemiology Collaboration creatinine equation, ${ }^{12}$ with age in years, as follows:

$$
\begin{aligned}
\operatorname{eGFR}\left(\mathrm{mL} / \mathrm{min} / 1.73 \mathrm{~m}^{2}\right)= & \begin{array}{l}
144 \times(\mathrm{Scr} / 0.7)^{-0.329} \times 0.993^{\text {Age }} \\
(\text { female }, \mathrm{Scr} \leq 0.7 \mathrm{mg} / \mathrm{dL})
\end{array} \\
\operatorname{eGFR}\left(\mathrm{mL} / \mathrm{min} / 1.73 \mathrm{~m}^{2}\right)= & 144 \times(\mathrm{Scr} / 0.7)^{-1.209} \times 0.993^{\text {Age }} \\
& (\text { female }, \mathrm{Scr}>0.7 \mathrm{mg} / \mathrm{dL}) \\
\operatorname{eGFR}\left(\mathrm{mL} / \mathrm{min} / 1.73 \mathrm{~m}^{2}\right)= & 141 \times(\mathrm{Scr} / 0.9)^{-0.411} \times 0.993^{\text {Age }} \\
& (\text { male, } \mathrm{Scr} \leq 0.9 \mathrm{mg} / \mathrm{dL}) \\
\operatorname{eGFR}\left(\mathrm{mL} / \mathrm{min} / 1.73 \mathrm{~m}^{2}\right)= & 141 \times(\mathrm{Scr} / 0.9)^{-1.209} \times 0.993^{\text {Age }} \\
& (\text { male }, \mathrm{Scr}>0.9 \mathrm{mg} / \mathrm{dL})
\end{aligned}
$$

\section{Study protocol}

The study protocol consists of three main steps (figure 1): screening and enrolment, baseline visit, and follow-up visits.

\section{Screening and enrolment}

The screening of potential subjects is conducted by nephrologists at each clinical centre to assess the eligibility for the study according to the inclusion and exclusion 


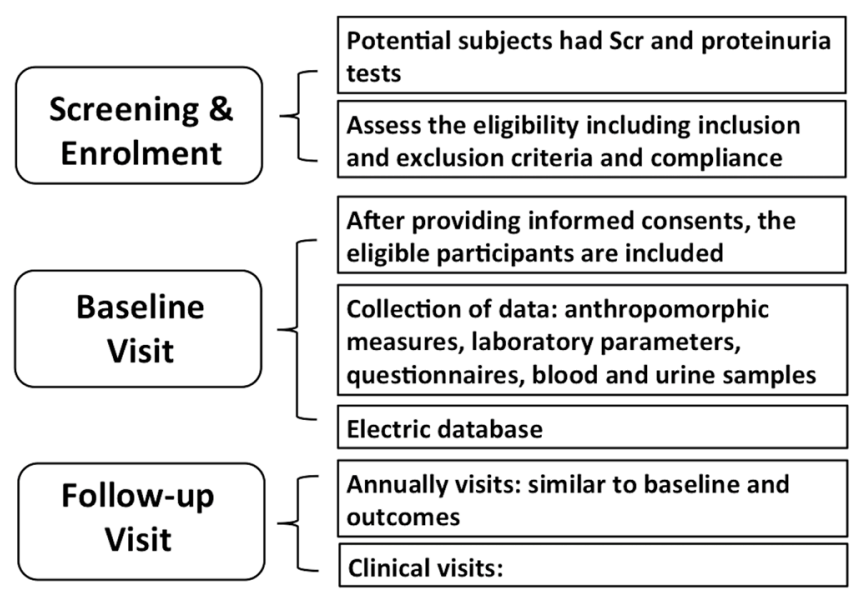

Figure 1 Flow chart of study design and procedures. eGFR, estimated glomerular filtration rate; Scr, serum creatinine.

criteria. When eligible, subjects signed informed consent forms before officially entering the study.

\section{Baseline visit}

On acceptance into the study, demographic information, laboratory parameters, anthropomorphic measures and questionnaires are collected from each patient.

Detailed demographic and clinical data include age, gender, ethnicity, residence, education, marital status, occupational status, income, housing, alcohol intake, smoking history, clinical aetiology of CKD (if known), comorbidities, medical history and use of medication.

Anthropomorphic measures include height, weight, waist circumference, hip and mid-arm circumference, triceps skinfold thickness, blood pressure, heart rate, handgrip strength and $5 \mathrm{~m}$ measured walk.

Laboratory items include full blood count, blood chemistry, haemoglobin A1c test for diabetes, routine and 24-hour urine, medical imaging, and others (table 1). There are some other special tests, such as pulse wave velocity and ankle-brachial index, that could not be conducted at all clinical centres, but will be performed in some centres as ancillary research.
Questionnaires on cognitive function, depressive symptoms, risk of malnutrition, functional and physical impairment, and health-related quality of life (HRQOL) are also collected (described below).

\section{Assessments based on questionnaires Cognitive function}

The Montreal Cognitive Assessment (MoCA) is an evaluation scale used to screen for mild cognitive impairment $(\mathrm{MCI})^{13}$ in elderly adults ${ }^{14}$ but is not valid for illiterate or those with little education. ${ }^{15} 16$ Thus, a revised MoCA test, the MoCA basic (MoCA-B), was developed. ${ }^{17}$ Given that China has a large proportion of elderly adults with little education, we chose the Chinese version of the MoCA-B (MoCA-BC) to screen for MCI. The MoCA-BC assesses nine cognitive domains: executive function, language, orientation, calculation, conceptual thinking, memory, visual perception, attention and concentration. The MoCA-BC is a screening tool that has been proven reliable in detecting MCI across all educational levels in Chinese elderly adults, with high acceptance and good reliability. ${ }^{18}$

\section{Psychological measures}

Symptoms of depression are assessed using the 15-item Geriatric Depression Scale (GDS-15), a reliable and well-validated measure of depressive symptoms in elderly patients. ${ }^{19}$ It has been validated for use in local Chinese, Malay and Indian subjects. ${ }^{20} 21$ This self-administered questionnaire contains 15 yes/no questions. Scores range from 0 to 15 , and higher scores indicate more severe depression.

\section{Risk of malnutrition}

Risk of malnutrition was measured according to the Malnutrition Inflammation Score (MIS), which was developed for patients on maintenance haemodialysis. ${ }^{22}$ It contains 10 items derived from medical history, physical examinations, body mass index and laboratory parameters. Each component is scored from 0 (normal) to 3 (severely abnormal). It has been reported that a revised MIS appears to be a useful tool to assess nutritional states

\section{Table 1 Baseline laboratory items/parameters for enrolled participants}

Full blood count Red blood cell, white blood cell, haemoglobin, neutrophilic granulocyte percentage, platelet count, red blood cell distribution width

\begin{tabular}{ll} 
Chemistry & $\begin{array}{l}\text { Scr, uric acid, albumin, BUN, carbon dioxide, glucose, serum potassium, calcium, phosphate, serum sodium, } \\
\text { chloride, prealbumin, transferrin, total iron binding capacity, cholinesterase, homocysteine, lipid panel }\end{array}$ \\
HbA1c & HbA1c (for diabetes) \\
Routine urine & Urine dipstick \\
24-Hour urine & Urine protein, electrolytes \\
Other items & Intact PTH, cystatin C, hs-CRP, ACR \\
Imaging & ECG, carotid artery ultrasound, echocardiogram, ABPM, BIA, lateral abdominal radiograph \\
\hline
\end{tabular}

*Lipid panel includes total cholesterol, triglycerides, high-density lipoprotein and low-density lipoprotein cholesterols.

ABPM, ambulatory blood pressure monitoring; ACR, albumin to creatinine ratio; BIA, bioelectrical impedance analysis; BUN, blood urea nitrogen; HbA1c, haemoglobin A1c; hs-CRP, high-sensitivity C reactive protein; PTH, parathyroid hormone; Scr, serum creatinine. 
Table 2 Items and procedures of the study at baseline and each follow-up

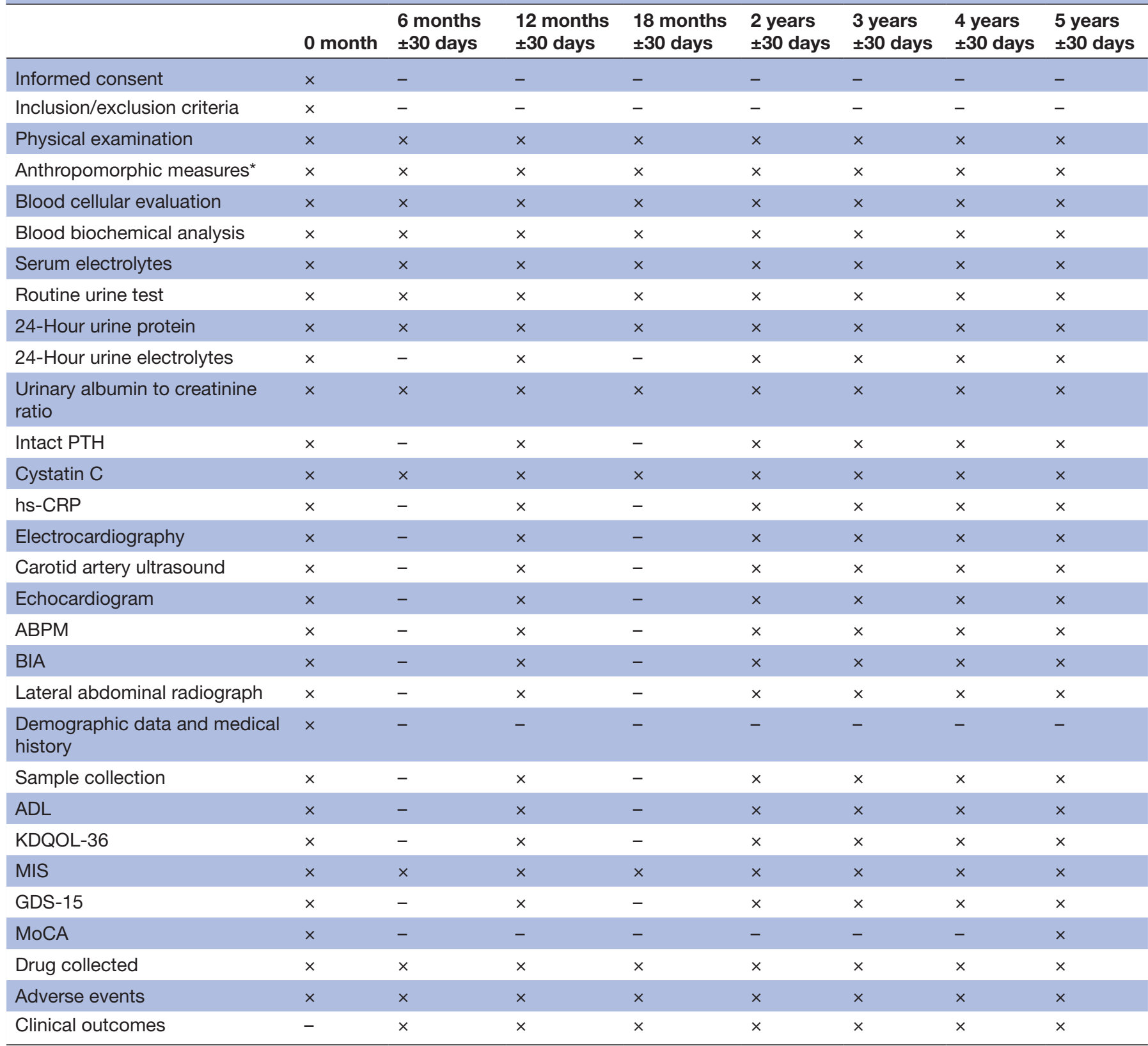

$\mathrm{x}$, item that will be collected.

-, item that will not be collected.

${ }^{*}$ Anthropomorphic measures: height, weight, waist circumference, hip circumference, mid-arm circumference, triceps skinfold thickness, blood pressure, heart rate, handgrip strength and $5 \mathrm{~m}$ measured walk.

ABPM, ambulatory blood pressure monitoring; ADL, Activity of Daily Living scale; BIA, bioelectrical impedance analysis; GDS, Geriatric Depression Scale; hs-CRP, high-sensitivity C reactive protein; KDQOL-36, Kidney Disease Quality of Life-36; MIS, malnutrition inflammation score; MoCA, Montreal Cognitive Assessment; PTH, parathyroid hormone.

and the essence of the protein-energy wasting (PEW), and to predict mortality risk in non-dialysed patients with CKD. ${ }^{23}$ In the validated MIS scale, dialysis vintage (ie, length of time on dialysis) was excluded from the score. Comorbidity was scored as follows: 0 , no other medical illnesses; 1 , mild comorbidity; 2 , moderate comorbidity (including one of the major comorbid conditions); and $3, \geq 2$ major comorbid conditions. In the present study, major comorbid conditions include congestive heart failure class III or IV, severe coronary artery diseases, moderate-to-severe chronic obstructive pulmonary disease or major neurological sequels. Serum transferrin was used instead of total iron binding capacity.

Assessment of functional disability

Functional and physical impairments are assessed using the Activity of Daily Living (ADL) and Lawton Instrumental Activities of Daily Living (IADL) scales. The ADL scale assesses self-maintaining activities of daily living, including feeding, dressing, grooming, transferring, 
mobility, stairs, and toilet use including bowel and bladder, and bathing. ${ }^{24}$ The IADL scale evaluates complex activities, including performance in transportation, preparing meals, taking medicine, housework, laundry, shopping, using telephone, managing finance and staying alone at home. Each item within the activities domains is scored from 0 to 4 .

\section{Quality of life}

Health-related quality of life (HRQOL) is assessed using the Kidney Disease Quality of Life-36 (KDQOL-36) instrument. ${ }^{25}$ KDQOL-36 was introduced in 1994 and is specific to kidney disease. The five subscales of the KDQOL-36 are mental component summary, physical component summary, burden of kidney disease, effects of kidney disease, and symptoms and problems of kidney disease. ${ }^{26}$ Low HRQOL is defined as a KDQOL-36 baseline score that is $>1 \mathrm{SD}$ below the mean.

\section{Follow-up visit}

Subsequent to the baseline evaluation, follow-up visits will be conducted over the study period to monitor study outcomes, update contact information and repeat the baseline assessments (table 2). All efforts are made to prevent dropout from the programme.

\section{Blood and urine samples collection}

Blood specimen, morning urine and 24-hour urine samples were collected at the baseline assessment and will be collected at the follow-ups. After simple processing, the specimens are frozen at $-80^{\circ} \mathrm{C}$ at each participating centre, and then transported to the central laboratory by cold chain every 3 months for future use.

With these specimens, we intend to explore for novel biomarkers that reflect CKD progression and develop predictive models to identify a subgroup population among elderly persons with CKD who are at high risk. Examples of biomarkers to explore include vascular endothelial progenitor cells, klotho protein, markers of muscle consumption, the senescence index silent information regulator $\mathrm{T} 1$ and telomere length.

\section{Study outcomes}

The primary outcomes of this study are all-cause mortality incidence of ESRD (ie, GFR $<15 \mathrm{~mL} / \mathrm{min} / 1.73 \mathrm{~m}^{2}$, or start of dialysis or renal transplantation) and loss of renal function ( $\geq 40 \%$ decline in GFR from baseline), with GFR confirmed at intervals of no less than 4 weeks.

The secondary endpoints include acute myocardial infarction, hospitalisation for congestive heart failure or unstable angina, cerebrovascular events (intracranial haemorrhage, subarachnoid haemorrhage, ischaemic stroke), and peripheral arterial disease. Acute myocardial infarction is defined as elevated levels of cardiac enzymes accompanied by symptoms such as a sudden painful sensation of pressure in the chest, profuse perspiration, nausea and vomiting, changes on ECG compatible with ischaemia or infarction, ${ }^{27}$ or new fixed perfusion abnormalities with corroborating wall motion.
Congestive heart failure is defined as hospitalisation for new or abrupt worsening of signs and symptoms and signs combined with falling cardiac output. Unstable angina is considered $>10 \mathrm{~min}$ of ischaemic symptoms that cannot be relieved by nitrate drugs, combined with new or aggravated ECG changes or imaging evidence of myocardial ischaemia, without changes of myocardial necrosis markers. Cerebrovascular events are diagnosed based on symptoms and changes of cranial CT scan or head MRI. Peripheral vascular disease is defined as amputation resulting from vascular disease or peripheral surgical or percutaneous revascularisation, or amputation resulting from vascular disease.

Suspected endpoint events will be determined by an independent assessment committee. In addition, an electronic database will be established, through which the Chinese PLA General Hospital could periodically supervise all participants to ensure complete information and input of outcome events. The determination of outcomes will also be supplemented by reports from patient proxies, hospital medical records and death certificates.

\section{Data analysis plan}

Continuous variables will be presented as mean \pm SD. Normally distributed variables will be compared using the Student's t-test. Non-parametric variables will be assessed by Mann-Whitney U test. Categorical variables will be presented as percentages and compared using the $\chi^{2}$ test.

There are several study endpoints, such as failure times including ESRD, reduction in eGFR and mortality. Kaplan-Meier curves, log-rank tests and the multivariate Cox proportional hazards analysis will be used to evaluate the associations between various baseline variables and study outcomes. ${ }^{28}$

Subgroup analyses will be conducted across different baseline eGFR level, proteinuria level and CKD aetiology, if needed. Receiver operating characteristic (ROC) curves will be used to evaluate the outcome predictability of baseline parameters by comparing the areas under the ROC curve, and to set the cut-offs of baseline parameters to predict renal outcomes in different subgroups.

Analysis of variables with repeated measures, such as eGFR, will use standard mixed effects growth curve models supplemented by generalised estimating equations. The characteristics of subjects without complete follow-up will also be analysed, and factors associated with dropout will be analysed.

A P value $<0.05$ will be considered statistically significant.

\section{Sample size}

The sample size for this study was calculated at 3000 participants with the following reasoning: We selected all-cause mortality as the primary study outcomes to calculate the sample size. Some studies have reported the mortality of older patients with CKD. Among 828 individuals older than 65 years, with eGFR $<60 \mathrm{~mL} / \mathrm{min}$ per $1.73 \mathrm{~m}^{2}$, there were 283 deaths $(34 \%)$ over 5 years. ${ }^{29}$ Another study revealed that the mortality rate was 
$125 / 1000$ patient-years and the ESRD rate was 63/1000 patient-years during a median follow-up of 3.3 years. ${ }^{30}$

On the basis of previous validation data and our own experience, we estimated that the incidence rate of mortality at 60 months of follow-up was at $\geq 10 \%$. There are 20-25 variables that may affect prognosis, and there should be at least 10 endpoints per variable. Thus we anticipate that recruitment of $\geq 3000$ participants with 200-250 end events will ensure adequate statistical power.

\section{Ethics and dissemination}

\section{Ethical approval}

All the participants gave written informed consent before recruitment. The study is non-invasive and imposes no significant risks to participants. Participants can withdraw from the study at any point with no effect on their clinical care. All data are anonymous and managed confidentially and anonymously.

\section{Dissemination}

The findings of the study will be published in peer-reviewed journals and will be presented at national or international conferences.

\section{DISCUSSION}

The ageing of the general population means that elderly people now account for a much greater proportion of patients with, or at risk of, CKD. It is reported that CKD affects $30 \%$ of adults older than 70 years and $50 \%$ of adults older than 80 years. ${ }^{31}$ The increasing prevalence of CKD in older adults highlights the need for more discerning medical management.

Previous findings indicate that age is an important effect modifier of the course of CKD. One large-scale study showed that, among patients of all ages with CKD, eGFR inversely correlated with both ESRD and mortality. However, at a given level of eGFR, compared with younger patients, the elderly had higher rates of death and lower rates of ESRD. ${ }^{32}$ These findings suggest that a uniform age-neutral approach is not appropriate for the management of elderly patients with CKD. In younger patients, a low eGFR is likely to result from a single disease affecting the kidney and thus may better predict renal outcomes. But in older patients, low eGFR appears to be a marker for various age-related coexisting conditions and a predictor of global health outcomes (eg, mortality), rather than specific renal outcomes.

Much heterogeneity exists among older adults of a similar age and eGFR regarding coexisting conditions and geriatric syndromes. This complicates CKD management and limits the utility of a single approach that may work well in younger populations. For example, a study from the USA showed that, for adults $\geq 75$ years of age, the presence of multiple non-disease-specific problems was associated with lower eGFR and higher ACR levels, where non-disease-specific problems included cognitive impairment, depressive symptoms, exhaustion, falls, impaired mobility and polypharmacy. Furthermore, the risk of death, hospitalisation and emergency department visits also correlated with the number of non-disease-specific problems. This suggests that the identification of non-disease-specific problems may provide credible risk factors of mortality, independent of kidney function among older patients with CKD. ${ }^{33}$ In our present study, we focus on the following five non-disease-specific problems as part of geriatric assessment: cognitive impairment, depressive symptoms, nutritional status, impaired mobility and quality of life.

The prevalence of CKD increases with age. Similarly, cognitive impairment is more common in the elderly. Several studies have investigated the association between CKD and cognitive impairment. The Cardiovascular Health Cognition Study ${ }^{34}$ reported a $37 \%$ increased risk of incident dementia in elderly individuals with an elevated Scr concentration. Another cross-sectional study of 1015 women with a mean age of $66.7 \pm 6.4$ years showed that eGFR was associated with performance on tests of global cognitive function, executive function, language and memory. Individuals with an eGFR $<30 \mathrm{~mL} / \mathrm{min} / 1.73 \mathrm{~m}^{2}$ were fivefold more likely to have cognitive impairment than those with an eGFR $<60 \mathrm{~mL} / \mathrm{min} / 1.73 \mathrm{~m}^{2}{ }^{35}$ The mechanisms that link CKD with cognitive impairment may include anaemia, oxidative stress, lipid and homocysteine metabolic dysregulation, and increased levels of inflammatory cytokines. There is some evidence that cognitive impairment predicts death and disability among the general elderly population ${ }^{3637}$ and dialysis patients. ${ }^{38}$ However, in elderly patients with predialysis CKD, little is known about whether cognitive impairment is associated with poor outcomes.

Depression is another common phenomenon in patients with late-stage CKD and ESRD. The prevalence of major depression and depressive symptoms varies from $15 \%$ to $45 \%$ in patients with ESRD or receiving dialysis. ${ }^{39} 40$ In several cross-sectional studies, ${ }^{41}{ }^{42}$ those with severe CKD (ie, with an eGFR $<30 \mathrm{~mL} / \mathrm{min} / 1.73 \mathrm{~m}^{2}$ ) had more depressive symptoms than those with eGFR $\geq 60 \mathrm{~mL} /$ $\min / 1.73 \mathrm{~m}^{2}$. Data have also shown that depression contributes to adverse outcomes in patients with ESRD, including higher risks of mortality, hospitalisation and cardiovascular events. ${ }^{43}{ }^{44}$ However, the number of population-based and longitudinal studies on depression in elderly patients with predialysis CKD is limited. ${ }^{45} 46$ The present study intends to include in its analysis the baseline prevalence of depression and its association with adverse outcomes at follow-up. We hypothesised that depressive symptoms among the elderly population with CKD may be related with cognitive impairment, poorer quality of life and increased mortality.

PEW is prevalent in dialysis patients and in patients with predialysis $\mathrm{CKD},{ }^{47}{ }^{48}$ and is associated with high mortality rates. ${ }^{49}$ Several indicators of PEW may predict cardiovascular events and mortality, such as serum albumin, prealbumin levels, subjective global assessment score and handgrip strength. ${ }^{30}$ One study of 1220 men 
with non-dialysis-dependent CKD showed that mortality was positively associated with white blood cell count, and negatively associated with albumin and lymphocyte percentage. Handgrip strength was also found to be a reliable nutritional marker in dialysis patients, and low handgrip strength predicted mortality. ${ }^{5}$

Malnutrition and inflammation are highly prevalent conditions in patients with CKD and intimately linked with PEW. The severity of malnutrition and inflammation can be assessed by the MIS scale. For example, during a follow-up of a median of 30 months (18-37 months), patients with higher MIS were at increased risk of mortality in both crude and adjusted Cox models, and $\mathrm{PEW}$ is more common in older patients. ${ }^{52} 53$ However, there is less information on the prevalence and magnitude of PEW in elderly patients with CKD, and the validity of PEW parameters as renal outcome predictors for this population has not been established. In the present study, the prevalence of PEW in older patients with CKD will be determined, and an association between the indicators of PEW and clinical outcomes will be investigated.

Patients who are under dialysis treatment have reduced levels of physical functioning. This and low physical activity predict poor outcomes. ${ }^{54}$ It is well known that older adults experience limitations in physical performance, which are associated with disability, healthcare utilisation and mortality ${ }^{55-57}$ But relatively little is known about the disabilities of elderly patients with predialysis CKD. A cross-sectional survey demonstrated that both mild and moderate CKD were associated with higher prevalence of disability. Age and other comorbid conditions account for most, but not all, of these associations, particularly among older adults. But the cross-sectional design of this study did not allow the inference of a causal relationship between disability and clinical outcomes such as death and decline in renal function. ${ }^{58}$ Thus, in the present study we aim to assess physical function and functional activity among elderly patients with predialysis CKD, and further investigate an association of disability and outcomes.

Persons with ESRD have poorer HRQOL compared with the general population, ${ }^{59}$ and poor HRQOL is associated with a higher risk of death and hospitalisation. ${ }^{6061}$ However, there have been few studies that investigated the relationship between diminished HRQOL and adverse outcomes among patients with predialysis CKD ${ }^{62}$ In the African American Study of Kidney Disease and Hypertension study, low baseline physical and mental HRQOL was associated with a higher risk of cardiovascular events and death, but not associated with CKD progression. ${ }^{63}$ In another study of 3837 participants with mild to severe CKD, the risk of cardiovascular events and death was independently associated with low physical component summary, effects and symptoms subscales, and risk of death was independently associated with low mental component summary subscale. However, none of the subscale scores were associated with CKD progression. ${ }^{64}$
Because of comorbidity and side effects of treatment, older patients with CKD are more likely to have poor HRQOL. HRQOL should be recognised as an important parameter of patient-centred care in elderly patients with CKD. In the present study, the KDQOL-36 will be used to analyse the demographic and clinical factors associated with low baseline HRQOL, and examine the association between baseline HRQOL and the risk of CKD progression, cardiovascular events and death.

A series of measures were included to minimise potential variation in results among the sites: (1) The relevant staff member of each centre was trained before the start of the study. (2) A manual of procedure was drawn up and distributed to each investigator, and detailed instructions are displayed in a uniform and standard manner. (3) An electronic database was established, and each site was asked to enter the data in a timely manner. (4) The quality of data is under the supervision of a professional research organisation composed of senior nephrologists, biostatisticians and an epidemiologist. Every month, $5 \%-10 \%$ of the cases from each participating site are selected randomly, and the validity of the data and questionnaires is checked. Unqualified data are returned to the investigators and recollected or refilled. Investigators will undergo repeated training, as necessary.

This study has some limitations. We recruited subjects based on a single measure of Scr and/or albuminuria without a 3-month interval. Second, there is no fully established eGFR equation to evaluate kidney function in the elderly. Third, there are potential selection bias or confounder effects because the study cohort is from tertiary hospitals instead of communities. We also excluded participants with severe impaired function, who were unable to complete the study procedure even if assisted. Therefore, the generalisability of the results to other populations may be compromised. Finally, the observational design precludes causality.

Collaborators Xue-qing Yu, Renal Division, Department of Medicine, The First Affiliated Hospital, Sun Yat-sen University, Guangzhou, China; Xiao-qiang Ding, Renal Division, Department of Medicine, Zhongshan Hospital, Fudan University, Shanghai, China; Wen-ge Li, Renal Division, Department of Medicine, China-Japan Friendship Hospital, Beijing, China; Hong-li Lin, Renal Division, Department of Medicine, The First Affiliated Hospital of Dalian Medical University, Dalian, China; Rong Wang, Renal Division, Department of Medicine, Shandong Provincial Hospital, Jinan, Beijing; Wen-hu Liu, Renal Division, Department of Medicine, Beijing Friendship Hospital, Capital Medical University, Beijing, China; Zhao-hui Ni, Renal Division, Department of Medicine, Renji Hospital Shanghai Jiaotong University School of Medicine, Shanghai, China; Ying Li, Renal Division, Department of Medicine, The Third Hospital of Hebei Medical University, Shijiazhuang, China; Li-ning Miao, Renal Division, Department of Medicine, Second Affiliated Hospital of Jilin University, Changchun, China; Guo-hua Ding, Renal Division, Department of Medicine, Renmin Hospital of Wuhan University, Wuhan, China; Fu-you Liu, Renal Division, Department of Medicine, The Second Xiangya Hospital of Central South University, Changsha, China; Ya-ni He, Renal Division, Department of Medicine, Daping Hospital, Third Military Medical University, Chongqing, China; Jing-ai Fang, Renal Division, Department of Medicine, First Hospital of Shanxi Medical University, Taiyuan, China; Feng-min Shao, Renal Division, Department of Medicine, Henan Provincial People's Hospital, Zhengzhou, China; Meng-hua Chen, Renal Division, Department of Medicine, General Hospital of Ningxia Medical University, Yinchuan, Ningxia, China; Xin-ling Liang, Renal Division, Department of Medicine, Guangdong General Hospital, Guangzhou, China; Hong-guang Zheng, Renal Division, Department of Medicine, General Hospital of Shenyang Military Command, 
Shenyang, China; Ai-hua Zhang, Renal Division, Department of Medicine, Peking University Third Hospital, Beijing, China; Cai-li Wang, Renal Division, Department of Medicine, The First Affiliated Clinical Hospital of Baotou Medical College, Baotou, China; Yong-ze Zhuang, Renal Division, Department of Medicine, Fuzhou General Hospital of Nanjing Military Command, Fuzhou, China; Zhi-yong Guo, Renal Division, Department of Medicine, Changhai Hospital, Second Military Medical University, Shanghai, China; Yue-yi Deng, Renal Division, Department of Medicine, LongHua Hospital Shanghai University of Traditional Chinese Medicine, Shanghai, China; Nian-song Wang, Renal Division, Department of Medicine, Shanghai Jiao Tong University, Affiliated to the Sixth People's Hospital, Shanghai, China; Qiang He, Renal Division, Department of Medicine, Zhejiang Provincial People's Hospital, Hangzhou, China; Ji-feng Sun, Renal Division, Department of Medicine, Tangdu Hospital, Fourth Military Medical University, Xi'an, China; Hong-wei Jiang, Renal Division, Department of Medicine, The First Affiliated Hospital, and College of Clinical Medicine of Henan University of Science and Technology, Luoyang, China; Xiao-ping Yang, Renal Division, Department of Medicine, The First Affiliated Hospital of the Medical College, Shihezi University, Shihezi, China; Yi-bin Yang, Renal Division, Department of Medicine, Affiliated Hospital of Zunyi Medical College, Zunyi, China; Jun Zhu, Renal Division, Department of Medicine, Chengdu Military General Hospital, Chengdu, China; Cheng-ai Deng, Renal Division, Department of Medicine, The First People's Hospital of Zhaotong, Zhaotong, China; Xin-zhou Zhang, Renal Division, Department of Medicine, Shenzhou People's Hospital, Shenzhen, China; Xiao-yan Wu, Renal Division, Department of Medicine, Zhongnan Hospital of Wuhan University, Wuhan, China; and Chen Lu, Renal Division, Department of Medicine, People's Hospital of Xinjiang Autonomous Region, Urumqi, China.

Contributors G-YC obtained the research funding and is the principal investigator of the study. SL and W-LW conceived and designed this study. SL drafted the manuscript. F-LZ, S-WD, X-FS and X-MC all participated in the final design of the study and revisions of this manuscript.

Funding This work was supported by the National Key Technology R\&D Program (2015BAl12B06, 2013BAI09B05, 2011BAl10B00), the 863 Program (2012AA02A512), the 973 Program (2013CB530800), the Science and Technology Project of Beijing (D131100004713003), and the NSFC (81171645).

Competing interests None declared.

Patient consent Obtained.

Ethics approval The ethics committees of Chinese PLA General Hospital and participating centers (no S2016-100-01).

Provenance and peer review Not commissioned; externally peer reviewed.

Open Access This is an Open Access article distributed in accordance with the Creative Commons Attribution Non Commercial (CC BY-NC 4.0) license, which permits others to distribute, remix, adapt, build upon this work non-commercially, and license their derivative works on different terms, provided the original work is properly cited and the use is non-commercial. See: http://creativecommons.org/ licenses/by-nc/4.0/

(c) Article author(s) (or their employer(s) unless otherwise stated in the text of the article) 2018. All rights reserved. No commercial use is permitted unless otherwise expressly granted.

\section{REFERENCES}

1. WHO. Interesting facts about aging. http://www.who.int/ageing/ about/facts/en/ (accessed 26 April 2014)

2. Tonelli M, Riella M. Chronic kidney disease and the ageing population. Lancet 2014;383:1278-9.

3. China Statistics Press. Tabulation on the 2010 population census of the people's Republic of China. 2015. Beijing, China: China Statistics Press, 2010.

4. Coresh J, Selvin E, Stevens LA, et al. Prevalence of chronic kidney disease in the United States. JAMA 2007;298:2038-47.

5. Keith DS, Nichols GA, Gullion CM, et al. Longitudinal follow-up and outcomes among a population with chronic kidney disease in a large managed care organization. Arch Intern Med 2004;164:659-63.

6. Tangri N, Kitsios GD, Inker LA, et al. Risk prediction models for patients with chronic kidney disease: a systematic review. Ann Intern Med 2013;158:596-603.

7. Bowling CB, Inker LA, Gutiérrez OM, et al. Age-specific associations of reduced estimated glomerular filtration rate with concurrent chronic kidney disease complications. Clin J Am Soc Nephrol 2011;6:2822-8.
8. Drawz PE, Babineau DC, Rahman M. Metabolic complications in elderly adults with chronic kidney disease. J Am Geriatr Soc 2012;60:310-5.

9. Hallan S, Astor B, Romundstad S, et al. Association of kidney function and albuminuria with cardiovascular mortality in older vs younger individuals: The HUNT II Study. Arch Intern Med 2007;167:2490-6.

10. Inker LA, Astor BC, Fox CH, et al. KDOQI US commentary on the $2012 \mathrm{KDIGO}$ clinical practice guideline for the evaluation and management of CKD. Am J Kidney Dis 2014;63:713-35.

11. Ma YC, Zuo L, Chen JH, et al. Modified glomerular filtration rate estimating equation for Chinese patients with chronic kidney disease. J Am Soc Nephrol 2006;17:2937-44.

12. Inker $\mathrm{LA}, \mathrm{Schmid} \mathrm{CH}$, Tighiouart $\mathrm{H}$, et al. Estimating glomerular filtration rate from serum creatinine and cystatin C. N Engl $J$ Med 2012;367:20-9.

13. Luis CA, Keegan AP, Mullan M. Cross validation of the montreal cognitive assessment in community dwelling older adults residing in the southeastern US. Int J Geriatr Psychiatry 2009;24:197-201.

14. Nasreddine ZS, Phillips NA, Bédirian V, et al. The Montreal Cognitive Assessment, MoCA: a brief screening tool for mild cognitive impairment. J Am Geriatr Soc 2005;53:695-9.

15. Zhou S, Zhu J, Zhang N, et al. The influence of education on Chinese version of Montreal cognitive assessment in detecting amnesic mild cognitive impairment among older people in a Beijing rural community. Scientific WorldJournal 2014;2014:1-7.

16. Freitas S, Simões MR, Alves L, et al. Montreal Cognitive Assessment: influence of sociodemographic and health variables. Arch Clin Neuropsychol 2012;27:165-75.

17. Julayanont $P$, Tangwongchai $S$, Hemrungrojn $S$, et al. The Montreal Cognitive Assessment-Basic: A Screening Tool for Mild Cognitive Impairment in Illiterate and low-educated elderly adults. J Am Geriatr Soc $2015 ; 63: 2550-4$.

18. Chen $\mathrm{KL}, \mathrm{Xu} Y, \mathrm{Chu} A Q$, et al. Validation of the chinese version of montreal cognitive assessment basic for screening mild cognitive impairment. J Am Geriatr Soc 2016;64:e285-e290.

19. Pomeroy IM, Clark CR, Philp I. The effectiveness of very short scales for depression screening in elderly medical patients. Int J Geriatr Psychiatry 2001;16:321-6.

20. Nyunt MSZ, Fones C, Niti M, et al. Criterion-based validity and reliability of the Geriatric Depression Screening Scale (GDS-15) in a large validation sample of community-living Asian older adults. Aging Ment Health 2009;13:376-82.

21. Broekman BFP, Nyunt SZ, Niti M, et al. Differential item functioning of the geriatric depression scale in an asian population. $J$ Affect Disord 2008;108:285-90.

22. Kalantar-Zadeh K, Kopple JD, Block G, et al. A malnutritioninflammation score is correlated with morbidity and mortality in maintenance hemodialysis patients. Am J Kidney Dis 2001;38:1251-63.

23. Amparo FC, Kamimura MA, Molnar MZ, et al. Diagnostic validation and prognostic significance of the malnutrition-inflammation score in nondialyzed chronic kidney disease patients. Nephrol Dial Transplant 2015;30:821-8

24. Lawton MP, Brody EM. Assessment of older people: selfmaintaining and instrumental activities of daily living. Gerontologist 1969;9:179-86.

25. Hays RD, Kallich JD, Mapes DL, et al. Development of the kidney disease quality of life (KDQOL) instrument. Qual Life Res 1994;3:329-38.

26. Ware J, Kosinski M, Keller SD. A 12-item short-form health survey: construction of scales and preliminary tests of reliability and validity. Med Care 1996;34:220-33.

27. Cooper RS, Simmons BE, Castaner A, et al. Left ventricular hypertrophy is associated with worse survival independent of ventricular function and number of coronary arteries severely narrowed. Am J Cardiol 1990;65:441-5.

28. Maldonado G, Greenland S. Simulation study of confounderselection strategies. Am J Epidemiol 1993;138:923-36.

29. Bansal N, Katz R, De Boer IH, et al. Development and validation of a model to predict 5 -year risk of death without ESRD among older adults with CKD. Clin J Am Soc Nephrol 2015;10:363-71.

30. Kovesdy CP, George SM, Anderson JE, et al. Outcome predictability of biomarkers of protein-energy wasting and inflammation in moderate and advanced chronic kidney disease. Am J Clin Nutr 2009;90:407-14.

31. U.S. Renal Data System. USRDS 2013 annual data report: atlas of chronic kidney disease and end-stage renal disease in the United States. National institutes of health, national institute of diabetes and digestive and kidney disease, 2013. 
32. O'Hare AM, Choi Al, Bertenthal D, et al. Age affects outcomes in chronic kidney disease. J Am Soc Nephrol 2007;18:2758-65.

33. Bowling CB, Booth JN, Gutiérrez OM, et al. Nondisease-specific problems and all-cause mortality among older adults with CKD: the REGARDS Study. Clin J Am Soc Nephrol 2014;9:1737-45.

34. Seliger SL, Siscovick DS, Stehman-Breen CO, et al. Moderate renal impairment and risk of dementia among older adults: the cardiovascular health cognition study. J Am Soc Nephrol 2004;15:1904-11.

35. Tsai YC, Hung CC, Hwang SJ, et al. Quality of life predicts risks of end-stage renal disease and mortality in patients with chronic kidney disease. Nephrol Dial Transplant 2010;25:1621-6.

36. Dewey ME, Saz P, Dementia SP. Dementia, cognitive impairment and mortality in persons aged 65 and over living in the community: a systematic review of the literature. Int $J$ Geriatr Psychiatry 2001;16:751-61.

37. Chertkow H. Mild cognitive impairment. Curr Opin Neurol 2002;15:401-7.

38. Griva K, Stygall J, Hankins M, et al. Cognitive impairment and 7-year mortality in dialysis patients. Am J Kidney Dis 2010;56:693-703

39. Lopes AA, Bragg J, Young E, et al. Dialysis Outcomes and Practice Patterns Study (DOPPS): depression as a predictor of mortalityand hospitalization among hemodialysis patients in the United States and Europe. Kidney Int 2002;62:199e-207.

40. Hedayati SS, Grambow SC, Szczech LA, et al. Physician-diagnosed depression as a correlate of hospitalizations in patients receiving long-term hemodialysis. Am J Kidney Dis 2005;46:642-9.

41. McClellan WM, Abramson J, Newsome B, et al. Physical and psychological burden of chronic kidney disease among older adults. Am J Nephrol 2010;31:309-17.

42. Ricardo AC, Fischer MJ, Peck A, et al. Depressive symptoms and chronic kidney disease: results from the National Health and Nutrition Examination Survey (NHANES) 2005-2006. Int Urol Nephrol 2010;42:1063-8.

43. Boulware LE, Liu Y, Fink NE, et al. Temporal relation among depression symptoms, cardiovascular disease events, and mortality in end-stage renal disease: contribution of reverse causality. Clin $J$ Am Soc Nephrol 2006;1:496-504.

44. Hedayati SS, Bosworth HB, Briley LP, et al. Death or hospitalization of patients on chronic hemodialysis is associated with a physicianbased diagnosis of depression. Kidney Int 2008;74:930-6.

45. Hedayati SS, Minhajuddin AT, Toto RD, et al. Prevalence of major depressive episode in CKD. Am J Kidney Dis 2009;54:424-32.

46. Andrade CP, Cruz MC, Urrutia M, et al. Evaluation of depressive symptoms in patients with chronic renal failure. $J$ Nephrol 2010;23:168e-74.

47. Mehrotra R, Kopple JD. Nutritional management of maintenance dialysis patients: why aren't we doing better? Annu Rev Nutr 2001;21:343-79.
48. Kalantar-Zadeh K, Ikizler TA, Block G, et al. Malnutrition-inflammation complex syndrome in dialysis patients: causes and consequences. Am J Kidney Dis 2003;42:864-81.

49. Fouque D, Kalantar-Zadeh K, Kopple J, et al. A proposed nomenclature and diagnostic criteria for protein-energy wasting in acute and chronic kidney disease. Kidney Int 2008;73:391-8.

50. Menon V, Greene T, Wang X, et al. C-reactive protein and albumin as predictors of all-cause and cardiovascular mortality in chronic kidney disease. Kidney Int 2005;68:766-72.

51. Wang AY, Sea MM, Ho ZS, Zs H, et al. Evaluation of handgrip strength as a nutritional marker and prognostic indicator in peritoneal dialysis patients. Am J Clin Nutr 2005;81:79-86.

52. Clark BC, Manini TM. Sarcopenia $=/=$ dynapenia. $J$ Gerontol A Biol Sci Med Sci 2008;63:829-34.

53. Morley JE, Abbatecola AM, Argiles JM, et al. Sarcopenia with limited mobility: an international consensus. J Am Med Dir Assoc 2011;12:403-9.

54. Stack AG, Molony DA, Rives T, et al. Association of physical activity with mortality in the US dialysis population. Am J Kidney Dis 2005;45:690-701.

55. Guralnik JM, Ferrucci L, Simonsick EM, et al. Lower-extremity function in persons over the age of 70 years as a predictor of subsequent disability. N Engl J Med 1995;332:556-62.

56. Guralnik JM, Ferrucci L, Pieper CF, et al. Lower extremity function and subsequent disability: consistency across studies, predictive models, and value of gait speed alone compared with the short physical performance battery. J Gerontol A Biol Sci Med Sci 2000;55:M221-31.

57. Studenski S, Perera S, Patel K, et al. Gait speed and survival in older adults. JAMA 2011;305:50-8.

58. Plantinga LC, Johansen K, Crews DC, et al. Association of CKD with disability in the United States. Am J Kidney Dis 2011;57:212-27.

59. Perlman RL, Finkelstein FO, Liu L, et al. Quality of life in chronic kidney disease (CKD): a cross-sectional analysis in the renal research institute-CKD study. Am J Kidney Dis 2005;45:658-66.

60. Mapes DL, Lopes AA, Satayathum S, et al. Health-related quality of life as a predictor of mortality and hospitalization: the Dialysis Outcomes and Practice Patterns Study (DOPPS). Kidney Int 2003;64:339-49.

61. Kalantar-Zadeh K, Kopple JD, Block G, et al. Association among SF36 quality of life measures and nutrition, hospitalization, and mortality in hemodialysis. J Am Soc Nephrol 2001;12:2797-806.

62. Kurella M, Yaffe K, Shlipak MG, et al. Chronic kidney disease and cognitive impairment in menopausal women. Am J Kidney Dis 2005; $45: 66-76$.

63. Porter A, Fischer MJ, Wang X, et al. Quality of life and outcomes in African Americans with CKD. J Am Soc Nephrol 2014;25:1849-55

64. Porter AC, Lash JP, Xie D, et al. Predictors and outcomes of healthrelated quality of life in adults with CKD. Clin J Am Soc Nephrol 2016;11:1154-62. 